\title{
ALL ALGEBRAS OF SPHERICAL FUNCTIONS DEFINED ON THE TWO-BY-TWO GENERAL LINEAR GROUP WITH ENTRIES IN A LOCALLY COMPACT p-ADIC FIELD ARE COMMUTATIVE ${ }^{1}$
}

\author{
ALLAN J. SILBERGER
}

1. Introduction. Let $\Omega$ be a locally compact $p$-adic field, with residue class field of odd characteristic, let $\vartheta$ denote the ring of integers in $\Omega$, and let $\tau$ generate the unique prime ideal in $\vartheta$, i.e. $\operatorname{ord}(\tau)=1$. Let $G=G L(2, \Omega)$, the group of nonsingular two-by-two matrices with entries in $\Omega$, and let $K=G L(2, \vartheta)$, the subgroup of integer matrices with unit determinant. $K$ is a maximal compact subgroup of $G$, and any maximal compact subgroup of $G$ is conjugate to $K$.

Let $u$ be an irreducible unitary representation of $K$ in a complex vector space $V_{u}$. Let $S_{u}$ denote the vector space consisting of functions $f$ on $G$ whose values are endomorphisms of $V_{u}$ and which satisfy $f\left(k g k^{\prime}\right)=u(k) f(g) u\left(k^{\prime}\right)$ for all $k, k^{\prime} \in K, g \in G$ (the product is composition of endomorphisms of $\left.V_{u}\right) . S_{u}$ is called the space of spherical functions of class $u$ on $G$. Set $f^{*}(g)=t \bar{f}\left(g^{-1}\right)$. Those elements of $S_{u}$ which are integrable (for the Haar measure) on $G\left(S_{u}^{1}\right)$ are seen to comprise a selfadjoint Banach algebra under convolution on $G$.

In this paper we shall prove that, for any $u, S_{\mathfrak{u}}^{1}$ is a commutative algebra. We remark that our result implies the commutativity of a class of Hecke algebras defined on certain two-by-two matrix groups; it also implies that $G L(2, \Omega)$ is a type I group (cf. [1] for an earlier proof that $G L(2, \Omega)$ is a type I group).

An earlier draft of this paper appeared as an appendix to the author's thesis at the Johns Hopkins University. He wishes to thank Professor Hisaaki Yoshizawa for conversations which led to the result presented here.

2. A reduction. Let $\epsilon$ be a nonsquare unit in $\vartheta$. Let $R$ be the subset of $K$ consisting of matrices of the form

$$
\left(\begin{array}{ll}
\alpha & \beta \\
\epsilon \beta & \alpha
\end{array}\right) .
$$

$R$ is a compact abelian group and is isomorphic to the group of units in the extension ring $\vartheta(\sqrt{ } \epsilon)$.

Received by the editors January 31, 1968.

1 Work partly supported by a National Science Foundation Grant. 
Let $\rho$ be a character of $R, A_{\rho}$ the convolution algebra of integrable functions on $G$ satisf ying the relation $f\left(r g r^{\prime}\right)=\rho\left(r r^{\prime}\right) f(g)$ for all $r, r^{\prime} \in R$, $g \in G$. It is easy to show that $S_{u}^{1}$ is isomorphic to a subalgebra of $A_{p}$ for any $\rho$ contained in the restriction to $R$ of $u$ (cf. $[2$, p. 173]). To prove $S_{u}^{1}$ is commutative for any $u$ we show that $A_{\rho}$ is commutative for any $\rho$.

3. The proof. To facilitate the proof of the commutativity of the algebras $A_{\rho}$ we introduce a "Cayley transform" for $G$.

For $g \in G$ set $C(g)=\gamma g \gamma^{-1}$, where

$$
\gamma=\left(\begin{array}{cc}
1 & -1 / \sqrt{ } \epsilon \\
\sqrt{ } \epsilon & 1
\end{array}\right) .
$$

$C$ is an isomorphism of $G$ with the group of all nonsingular matrices of the form

$$
\left(\begin{array}{rr}
a & b \\
-\epsilon b & a
\end{array}\right)
$$

where $a, b \in \Omega(\sqrt{ } \epsilon)$ and the bar denotes conjugation in $\Omega(\sqrt{ } \epsilon) . C(K)$ $=C(G) \cap G L(2, \vartheta(\sqrt{ } \epsilon)) ; C(R)$ is the set of diagonal matrices in $C(K)$. From now on we identify $C(G)$ and $G$.

In the transformed version of $G$ we consider two involutions. The mapping $g$ to $\bar{g}$. where $\bar{g}$ has entries conjugate to those of $g$, is an order-two automorphism of $G$. For

$$
g=\left(\begin{array}{rr}
a & b \\
-\epsilon \bar{b} & \bar{a}
\end{array}\right)
$$

$0 \neq \Delta(g)$, the determinant of $g$, set

$$
g^{\sigma}=\Delta^{-1}(g)\left(\begin{array}{rr}
\bar{a} & b \\
-\epsilon \bar{b} & a
\end{array}\right) \text {. }
$$

$\sigma$ is an order two antiautomorphism of $G$. Both involutions clearly leave a Haar measure on $G$ invariant.

We shall say $g$ is $\sigma$-symmetric if $g^{\sigma}=g \Delta^{-1}(g)$. This is the case if and only if diagonal entries of $g$ belong to $\Omega$. Note that $g^{\sigma}=g^{-1}$ if and only if $g$ is a diagonal matrix (a scalar times an element of $R$ ). We denote the $\sigma$-symmetric elements of $G$ by $S$.

LEMMA 1. (1) $G=S R$.

(2) $r s r^{\prime} \in S, s \in S, r, r^{\prime} \in R$ if and only if, diagonal entries of $s$ are zero or $r^{\prime}=\lambda r^{-1}, \lambda$ a scalar matrix with unit entries in $\vartheta$.

(3) For all $f \in A_{\rho}$ and all $s \in S, f(s)=f(\xi)$. 
Proof of (3) only. For any $a \in \Omega$, unit $b \in \vartheta(\sqrt{ } \epsilon)$, and integer $k$ we have

$$
\left(\begin{array}{ll}
b & 0 \\
0 & b
\end{array}\right)\left(\begin{array}{cc}
a & b \tau^{k} \\
-\epsilon b \tau^{k} & a
\end{array}\right)=\left(\begin{array}{cc}
a & b \tau^{k} \\
-\epsilon b \tau^{k} & a
\end{array}\right)\left(\begin{array}{ll}
b & 0 \\
0 & b
\end{array}\right)
$$

(3) follows immediately.

Next we induce an involution $\sigma$ on $A_{\rho}$ and show that the $\sigma$-symmetric elements of $A_{\rho}$ form a commutative real subalgebra.

Lemma 2. For $f \in A_{\rho}$, set $f^{\sigma}(g)=\bar{f}\left(g^{\sigma}\right)$. Then $f^{\sigma} \in A_{\rho}$ and the mapping $\sigma: A_{\rho} \rightarrow A_{\rho}$ is a complex-conjugate linear antiautomorphism.

Proof. $f \in A_{\rho}$ implies $f^{\sigma} \in A_{\rho}$, since $f^{\sigma}\left(r g r^{\prime}\right)=\bar{f}\left(\left(r g r^{\prime}\right)^{\sigma}\right)=f\left(r^{\prime} g^{\sigma} r^{\sigma}\right)$ $=\bar{f}\left(r^{\prime-1} g^{\sigma} r^{-1}\right)=\rho\left(r r^{\prime}\right) \bar{f}\left(g^{\sigma}\right)$, for any $r, r^{\prime} \in R, g \in G$. That $\sigma$ is complexconjugate linear is clear. To see that $\sigma$ is an antiautomorphism observe that

$$
\begin{aligned}
(f * h)^{\sigma}(g) & =\int_{\sigma} \bar{f}(x) \bar{h}\left(x^{-1} g^{\sigma}\right) d x=\int_{\sigma} \bar{f}\left(g^{\sigma} u^{-1}\right) \bar{h}(u) d u \\
& =\int_{\sigma} \bar{h}\left(u^{\sigma}\right) \bar{f}\left(g^{\sigma}\left(u^{-1}\right)^{\sigma}\right) d u=\int_{\sigma} h^{\sigma}(u) f^{\sigma}\left(u^{-1} g\right) d u=h^{\sigma} * f^{\sigma}(g) .
\end{aligned}
$$

Call $f \in A_{\rho} \sigma$-symmetric if $f^{\sigma}(g)=f(g)$ for all $g \in G$.

LEMMA 3. (1) $f \in A_{\rho}$ is $\sigma$-symmetric if and only if $f(g)=\bar{f}\left(\Delta^{-1}(g) \bar{g}\right)$.

(2) The $\sigma$-symmetric elements of $A_{\rho}$ comprise a commulative real subalgebra of $A_{\rho}$.

Proof. (1) $f^{\sigma}(g)=f^{\sigma}(s r)=\bar{f}\left(r^{\sigma} s^{\sigma}\right)=\bar{f}\left(r^{-1} \Delta^{-1}(s) \bar{s}\right)=\rho(r) \bar{f}\left(\Delta^{-1}(s) \bar{s}\right)$ $=\rho^{-1}(\bar{r}) \rho(\Delta(r)) \bar{f}\left(\Delta^{-1}(s) \bar{s}\right)=\bar{f}\left(\Delta^{-1}(g) \bar{g}\right)$.

(2) Clearly, real linear combinations of $\sigma$-symmetric elements of $A_{\rho}$ are $\sigma$-symmetric. We must show both that $f * h(s)=\bar{f} * \bar{h}\left(\Delta^{-1}(s) \bar{s}\right)$ and that $f * h(s)=h * f(s)$ for any $f$ and $h \sigma$-symmetric and any $s \in S$. We have

$$
\begin{aligned}
f * h(s) & =\int_{G} f(g) h\left(g^{-1} s\right) d g \\
& =\int_{G} \bar{f}\left(\Delta^{-1}(g) \bar{g}\right) \bar{h}\left(\Delta(g) \bar{g}^{-1} \Delta^{-1}(s) \bar{s}\right) d g \\
& =\int_{G} \bar{f}(x) \bar{h}\left(x^{-1} \Delta^{-1}(s) \bar{s}\right) d x=\bar{f} * \bar{h}\left(\Delta^{-1}(s) \bar{s}\right) ;
\end{aligned}
$$

and $f * h(s)=(f * h)^{\sigma}(s)=h^{*} * f^{\sigma}(s)=h * f(s)$. 
Proposition. $A_{\rho}$ is commutative.

Proof. Given $f \in A_{\rho}$, we may write $f=f_{1}+i f_{2}$, where $f_{i}$ is $\sigma$-symmetric, $i=1$ or 2 . Indeed, $f_{1}=\left(f+f^{\sigma}\right) / 2$ and $f_{2}=\left(f-f^{\sigma}\right) / 2 i$. The commutativity of $A_{\rho}$ follows immediately from Lemma 3 .

4. Extension of results to the projective general linear group. Our results extend immediately, by a lifting argument, to the case of $G=P G L(2, \Omega)$, the factor group of $G L(2, \Omega)$ by the scalar matrices, and $K=P G L(2, \vartheta)$. In $P G L(2, \Omega)$ there is a second conjugacy class of maximal compact subgroups, a representative of which is generated by the projections to $P G L(2, \Omega)$ of the matrices

$$
\left\{k=\left(\begin{array}{ll}
k_{11} & k_{12} \\
\tau k_{21} & k_{22}
\end{array}\right): \begin{array}{l}
k_{i j} \in \vartheta, \quad 1 \leqq i, j \leqq 2 \\
\Delta^{-1}(k) \in \vartheta
\end{array}\right\} \cup\left\{\left(\begin{array}{ll}
0 & 1 \\
\tau & 0
\end{array}\right)\right\} .
$$

This group contains a compact abelian subgroup isomorphic to $\Omega^{\times}(\sqrt{ } \tau) / \Omega^{\times}$, the quotient of multiplicative groups. A variant of the argument of this paper extends the conclusions.

\section{REFERENCES}

1. A. A. Kirillov, Infinite-dimensional unitary representations of a second-order matrix group with elements in a locally compact field, Dokl. Akad. Nauk SSSR 150 (1963), 740-743=Soviet Math. Dokl. 4 (1963), 748-752.

2. F. I. Mautner, Spherical functions over p-adic fields. II, Amer. J. Math. 86 (1964), 171-200.

\section{Bowdoin College}

